\title{
Off-line Bangla Signature Verification: An Empirical Study
}

\author{
Srikanta $\mathrm{Pal}^{1}$, Alireza Alaei ${ }^{2}$, Umapada $\mathrm{Pal}^{3}$, Michael Blumenstein ${ }^{1}$ \\ ${ }^{1}$ School of Information and Communication Technology, Griffith University, Australia, \\ ${ }^{2}$ Laboratoire d'Informatique, Polytech'Tours, Université François-Rabelais, Tours, France \\ ${ }^{3}$ Computer Vision and Pattern Recognition Unit, Indian Statistical Institute, Kolkata, India. \\ Email: srikanta.pal@griffithuni.edu.au
}

\begin{abstract}
Among all of the biometric authentication systems, handwritten signatures are considered as the most legally and socially accepted attributes for personal verification. The objective of this paper is to present an empirical contribution towards the understanding of a threshold-based signature verification technique involving off-line Bangla (Bengali) signatures. Experiments on signature verification involving non-English signatures are an important consideration in the signature verification area. Only very few research works employing signatures of Indian script have been considered in the field of non-English signature verification. To fill this gap, a threshold-based scheme for verification considering off-line Bangla signatures is proposed. Some techniques such as undersampled bitmap, intersection/endpoint and directional chain code are employed for feature extraction. The Nearest Neighbour method is considered for classification. Furthermore, a Bangla signature database, which consists of $2400(100 \times 24)$ genuine signatures and $3000(100 \times 30)$ forgeries has been created and is employed for experimentation. We obtained a $15.57 \%$ Average Error Rate (AER) as the best verification result using directional chain code features employed in this research work.
\end{abstract}

\section{INTRODUCTION}

$\mathrm{T}$ ODAY, biometric technologies are increasingly being used to ensure identity verification or the authorisation of access to sensitive data. Among all of the biometric authentication systems, handwritten signatures have been accepted as an official means to verify personal identity for legal purposes on such documents as cheques, credit cards and wills [1].

The development of computer-aided handwritten signature verification systems has been ongoing for decades $[1]$. In $[1,13]$, a thorough survey of automatic handwritten signature verification and writer identification techniques was provided. Justino et al. [2] proposed an off-line signature verification system based on Hidden Markov Models (HMMs) to detect random, casual, and skilled forgeries. Jing Wen et al. [14] presented a feature extraction method based on the intensity of the coefficients of the Gabor transform. The image is subjected to a multichannel Gabor Transform, then the transformed Gabor image is divided equally into $\mathrm{N} 1 * \mathrm{~N} 2$ non-overlapping boxes. The angle features of the position of the maxima intensity of the Gabor Transform Coefficients are extracted. Ramachandra et al. [15] also proposed an offline signature authentication system using cross-validated graph matching. A bipartite graph is constructed from which a minimum cost-complete match is obtained. The Euclidean distance is calculated by the Hungarian method. A cross-validation principle is used to select reference signatures. Another signature verification method was presented by Mahar et al. [16]. Three kinds of features such as: Grid, Global, and Texture Feature Comparison are used for signature verification. When employing grid-based features, a signature image is divided into rectangular regions, and the ink distribution in each region is evaluated. In the global feature comparison, a number of features extracted globally from the whole signature are compared. The texture-based feature comparison is based on the co-occurrence matrices of the signature image. The Euclidian distance is used for offline signature verification.

In some recent work, Pal et al. [20] presented a signature verification system employing Hindi Signatures. The objective of this paper was to present an investigation of the performance of a signature verification system involving Hindi offline signatures. Hindi is a very popular language in India, written in Devnagari script. Encouraging results were obtained in this investigation. Recently, Pal et al. [8] presented a signature verification system based on a local language in India. In their paper, the performance of an offline signature verification system involving Bangla (Bengali) signatures, whose style is distinct from Western scripts, was investigated. The Gaussian Grid feature extraction technique was employed for feature extraction and Support Vector Machines (SVMs) were considered for classification. The Bangla signature database employed in those experiments consisted of 3000 forgeries and 2400 genuine signatures.

However, the techniques presented in the literature for signature verification of other languages such as English, Japanese, Chinese and Persian may not be suitable for the numerous Indic scripts and languages, which are officially in use in India and Bangladesh. To deal with the problem of multi-script signature verification in such an environment, one possible solution may be the identification of signatures in the first stage and then signature verification in the next stage. A few methods are proposed for signature identification of Bangla, Hindi, Chinese and English signatures [17] and a piece of work is also presented on Bangla signature verification [18]. Since Bangla signatures are mainly composed of a text portion, in this paper, an empirical study is performed for Bangla signature 
verification using different features with a particular focus on a threshold-based technique. Some Bangla signature samples are shown in Table I.

The remainder of this paper is organized as follows. The signature verification concept is described in Section II. Section III describes the importance of Bangla signature verification. The Bangla signature database developed for the current research is described in Section IV. Section V briefly describes the feature extraction technique employed. Details of the classifiers used are presented in Section VI. The experimental settings are presented in Section VII. Results and discussion are given in Section VIII. Finally, conclusions and future work are discussed in Section IX.

TABLE I

Signature SAMPLES OF BANGLA SCRIPT

\begin{tabular}{|c|c|}
\hline कीजू उक्य लr & onartom on smy \\
\hline mpमnन कूरू & 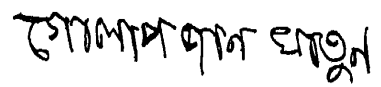 \\
\hline अंभूझान $6 \mathrm{~h}$ & 6 मnd $\$ 127$ \\
\hline
\end{tabular}

\section{SignATURE VERIFICATION CONCEPT}

In the area of personal authentication, signature verification is considered a very popular biometric method. Generally, off-line/on-line signature verification is formulated as a two-class classification problem where the classes are associated with a genuine signature set and a forged signature set. Usually two types of errors [21] are considered in a signature verification system: The False Rejection or Type-I error and the False Acceptance or TypeII error. These error types associate with two common types of error rates: False Rejection Rate (FRR), which is the percentage of genuine signatures misclassified as forgeries, and False Acceptance Rate (FAR) which is the percentage of forged signatures misclassified as genuine. A general signature verification approach is shown in Fig. 1.

The forgeries involved in handwritten signatures have been categorized based on their characteristic features. Three different types of forgeries are taken into account in the field of signature verification. According to Coetzer et al. [9], the three basic types of forged signatures are:

\section{A. Random forgery}

The signer uses the name of the author in his own style to create a forgery known as a simple or random forgery. It is done by a person who doesn't know the shape and structure of the original signature. This type of forgery is very easy to detect. In many cases, the forgeries are the forgers' own genuine signature.

\section{B. Simple forgery}

The forger knows the author's name and the script, but has no access to a sample of the signature.

\section{C. $\quad$ Skilled forgery}

The last type is the skilled forgery, performed by an expert person. The forger has a good knowledge about the original signature and signs with sufficient practice. Naturally it is more difficult to detect skilled forgeries than other forgeries.

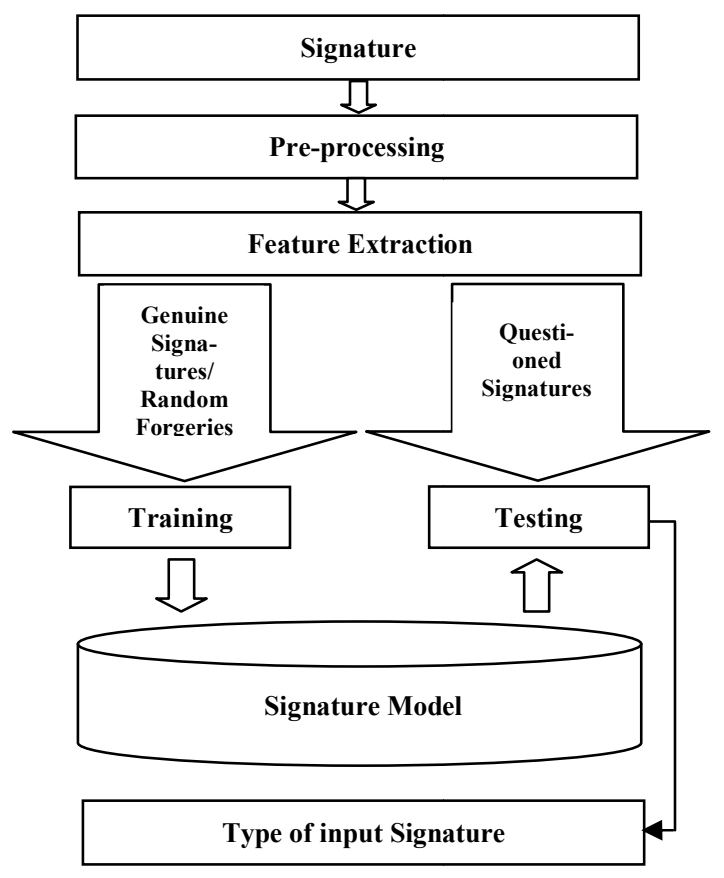

Fig. 1. Signature verification approach

\section{IMPORTANCE OF BANGLA SIGNATURE VERIFICATION}

A country having two or more scripts and languages is known as a multi-script and multi-lingual country. Most countries have only a single language but very few countries have more than one script for reading and writing purposes. However in India, there are officially 23 (Indian constitution accepted) languages and 11 different scripts. Generally, scripts are employed for writing languages down in a readable form. In such a multi-script and multi-lingual country like India, Bangla script, as one of the official languages of India, is not only used for writing/reading purposes but also for signing purposes.

Bangla signature verification is quite different with respect to the shape and structure of Bangla signature images. Most of the characters of Bangla hand-written words are touching. A distinctive horizontal line running along the top of the letters that links them together is present. Bengali script is less blocky and presents a more sinuous shape. Bangla signatures always contain textual components. For Bangla signatures, there is no such concept of an "initial signature" (first letter of the first name followed by a family name) which is seen frequently in English signatures.

To get the desired accuracy when dealing with pattern recognition problems, feature set selection takes on a vital role. It is sometimes difficult to choose the appropriate 
features because of the different characteristics of various scripts. The features used here (under-sampled bitmap, intersection/endpoint and directional chain code) are suitable for Bangla script with the characteristics previously described.

\section{SignATURE DATABASE}

Some notable properties of Bangla script and the Bangla signature dataset used for these experiments are described here.

\section{A. Properties of Bangla Script}

Bangla is the second most popular language in India. Bangla script is widely used for official reasons throughout West Bengal and Assam (two states of India). Other countries using Bangla include Bangladesh. It is estimated that there are about 300 million people using Bangla script. Bangla is an oriental script descended from Brahmi script [19]. In Bangla script, the writing direction is from left to right and does not have the concept of distinct letter cases. It is identifiable by a distinct horizontal line called the 'head line' that runs along the top of full letters and it connect all the letters together in a word. Bangla script has about fifty basic characters. These characters are presented in Fig. 2.

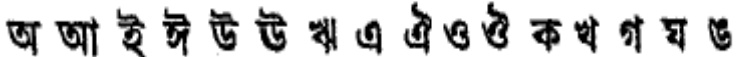

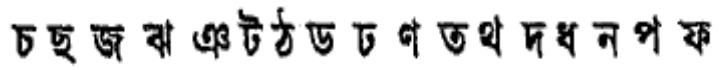 ব ए म य র ल य य म इ ए़ ए़ य : :}

Fig. 2. Basic characters of Bangla script

\section{B. Data Collection and Preparation}

Although automatic signature verification has been an active research area for several decades, and a few English language signature databases are available for research [11], there has been no publicly available signature database for Bangla, the second most popular Indian script. Moreover, research on Indian-based script signatures has gradually increased in recent times. Therefore, a Bangla signature corpus was created for this work.

The signatures were collected from different parts of the West Bengal State of India. The majority of the signatures were contributed by students. The remaining signatures were collected from seniors of the locality. This Bangla signature database consists of 100 sets (classes). In order to collect the genuine signatures corresponding to each individual, a collection form was designed. The form contained 24 boxes where the signatures could be written. From each individual, 24 genuine signatures were collected. A total number of 2400 genuine signatures were collected from 100 individuals. For each contributor, all genuine specimens were collected in a single day's writing session. In addition, skilled forged signatures were collected for this work. In order to produce the forgeries, the imitators were allowed to practice their forgeries as long as they wished with static images of genuine specimens. A total number of 3000 (30 signatures $\times 100$ individuals) forged signatures were collected from the writers. In summary, the Bangla signature database consists of 5400 signatures of which 2400 (24 signatures $\times 100$ individuals) signatures are genuine signatures and 3000 (30 signatures $\times 100$ individuals) signatures are forged signatures. Some genuine signature samples with their corresponding forgeries are shown in Table II. From Table II, it can be noted that in the Bangla signature database provided in this research work, the genuine and forged signatures are very similar. This property of the Bangla signature database makes the verification task very challenging.

TABLE II

EXAMPLES OF GENUINE AND FORGED SIGNATURES OF BANGLA SCRIPT

\begin{tabular}{|c|c|}
\hline Genuine Signatures & Forged Signatures \\
\hline 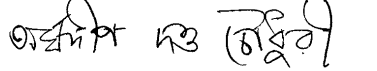 & 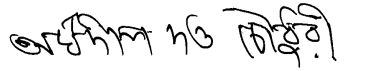 \\
\hline ऊभा बाल & अचना बाल \\
\hline 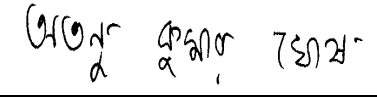 & orog खs aा! (b)|r \\
\hline 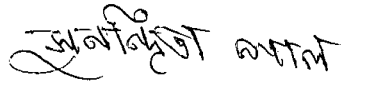 & स्नतनुंजवान \\
\hline
\end{tabular}

\section{FEATURE EXTRACTION}

Feature extraction is a vital step in any pattern recognition system. Different methods have been proposed in the offline signature verification literature to perform the extraction of features from signature images [12]. In this research work, three different feature extraction methods, used in the literature for document recognition, are studied. These features are chosen, since, they have shown their strength on text patterns [3-6] and Bangla signatures are mainly textual patterns. In the following, brief descriptions of three different feature sets used in this paper are discussed.

It is worth mentioning that very small components (mainly dots or noise) are eliminated from every signature image using a threshold based on the average size of connected components in the image to have a proper bounding box of the signature (Fig. 3(b)).

\section{A. Undersampled Bitmap Features}

Feature extraction based on undersampled bitmaps is a simple technique used in the literature by many researchers $[3,4]$ for recognition purposes. The undersampled bitmaps are formed by dividing each input image into a number of non-overlapping blocks of similar size. Then, the number of black pixels is counted in each block. This generates an input matrix with each element being an integer in the range 0 to the size of the non-overlapping block. Dividing these values by the size of the block, the values are normalized between 0 and 1 . The undersampling process reduces dimensionality of 
the features compared to the whole image size and provides invariance to small distortions and slant [4].

In our study, after binarizing the input image, a minimumbounding box of the input image is obtained (Fig. 3(b)). Then, for a better result and independence of features to size and position (invariant to scale and translation), the minimum-bounding box of the image is converted into a normal size of $200 \times 800$ pixels (Fig. 3(c)). This value is determined experimentally. To compute the undersampled bitmap features, the normalized image $(200 \times 800)$ is divided into 80 non-overlapping blocks of size $25 \times 80$ pixels (Fig. $3(\mathrm{c}))$. Then, the number of black pixels is counted in each block. This generates an input matrix of $8 \times 10$ with each element being an integer in the range 0 to $2000(25 \times 80)$. Dividing these values by the size of the block (2000), the values are normalized between 0 and 1 . Since, the normalized image is divided into 80 blocks, 80 features are obtained for each input signature.

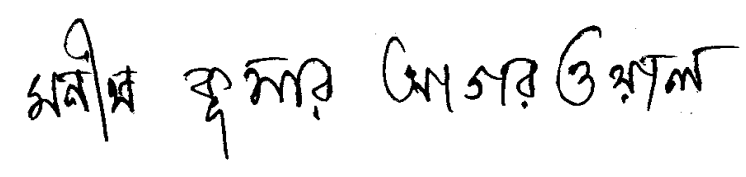

(a)

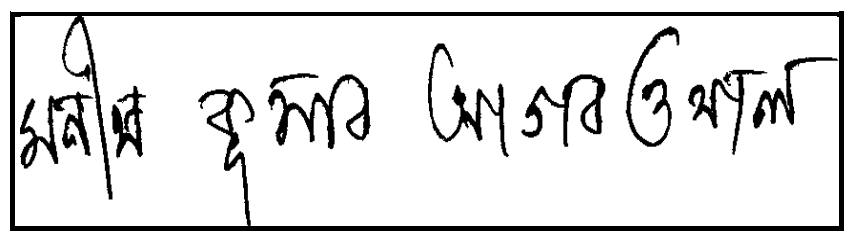

(b)

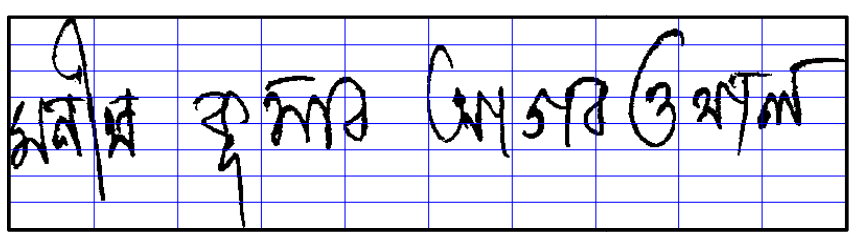

(c)

Fig. 3. (a) A Bangla signature sample, (b) Bounding box of the Bangla signature(after removing the small components), (c) Normalized image $(200 \times 800)$ and its non-overlapping window-map of size $25 \times 80$ on a normalized image.

\section{B. Intersection/Junction/End Points Features}

An intersection point is defined as a pixel point which has more than two neighbouring pixels with 8-connectivity, while an endpoint has exactly one neighbouring pixel. Intersection features are extracted from the thinned signature image, which is first normalized into $200 \times 800$ pixels. For the thinning process, the algorithm presented in [5] is utilized. The thinned signature image is then divided into 20 blocks each of size $100 \times 80$ pixels. For each block, the number of endpoints and intersection/junctions are found and counted separately. Thus, $40(20 \times 2)$ features from 20 constituent blocks of the signature image are computed, out of which the first 20 features represent the number of end points and the remaining 20 features represent the number of intersection/junction points. Intersection points and endpoints of a Bangla signature image are shown in Fig. 4.

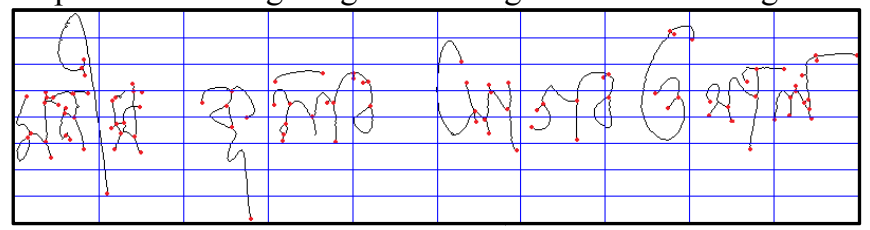

Fig. 4. Intersection points and endpoints are shown by red marks on a thinned signature image (for better visibility of the figure see the soft copy of the paper).

\section{Directional Chain Code Features}

The chain code direction information of the contour points of an input image have been used as features for different purposes, including numeral and character recognition [6]. Contour information can provide a good representation of signature shape. To compute directional chain code features, similar to the undersampled and intersection/end point feature extraction techniques, at first, the minimum bounding box of the input image is obtained and the minimum bounding box is normalized into $200 \times 800$ pixels. Using the normalized binary image, the contour points of the signature image are found based on the 8-connectivity formula (Fig. 6).

The image contour is scanned horizontally by keeping an overlapping window-map of size $27 \times 82$ (Fig. 6) on the image from the top-left most point to the down-right most point (80 overlapping blocks). For each overlapping block, the chain code frequencies for all 8 directions (Fig. 7) are computed. Instead of expressing the features in terms of 8 directions, we simplify the features into 4 directions (Fig. 8): (i) Horizontal direction code (directions 0 and 4), (ii) Vertical direction code (directions 2 and 6), (iii) Diagonal direction code (directions 1 and 5) and (iv) Off-diagonal direction code (directions 3 and 7). Thus, in each block, four features representing the frequencies of these four directions are obtained. As a result, for each image we obtain $320(80 \times 4)$ features from 80 blocks. The reason for choosing an overlapping window-map (one pixel from each side) instead of a non-overlapping window-map is to preserve the information between a window-map and its neighbouring blocks. Moreover, based on an experimental study we have extracted feature sets from non-overlapping as well as overlapping blocks, and overlapping blocks provided better performance.

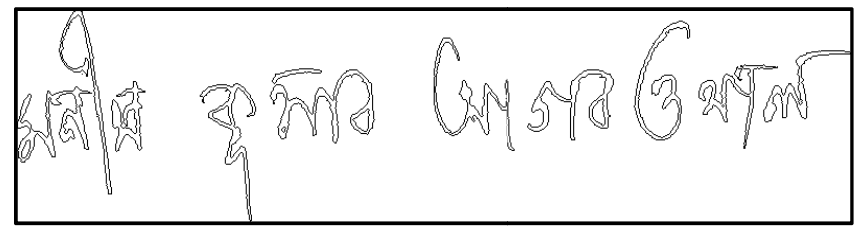

Fig. 5. Inner and outer contour of the Bangla signature shown in Fig. 3(c).

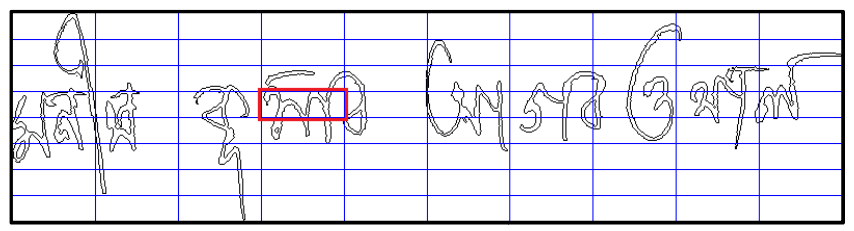

Fig. 6. An overlapping window-map of size $27 \times 82$ is shown in red on the contour image. 


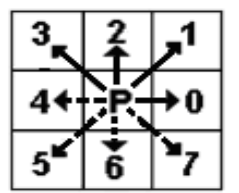

Fig. 7. Point $P$ and its 8 -direction codes.

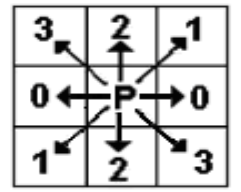

Fig. 8. Four directions obtained from 8 directions.

\section{Classifier Details}

Amongst the various methods of supervised statistical pattern recognition, the Nearest Neighbour (NN) rule achieves consistently high performance, without a priori assumptions about the distributions from which the training examples are drawn. A new sample is classified by calculating the distance to the nearest training case; the sign of that point then determines the classification of the sample. The distances can be calculated using one of the distance measures such as Euclidian, Mahalanobis and City-block. In this paper, the Euclidian distance measure is used for experimentation.

TABLE III

VERIFICATION PERFormance OF CONTOUR FeATURES ON THE BANGLA DATASET BASED ON DIFFERENT THRESHOLD VALUES.

\begin{tabular}{|l|c|c|c|}
\hline Threshold & $\begin{array}{c}\text { FAR } \\
(\%)\end{array}$ & $\begin{array}{c}\text { FRR } \\
(\mathbf{\%})\end{array}$ & $\begin{array}{c}\text { AER } \\
\mathbf{( \% )}\end{array}$ \\
\hline $0.8 *$ Average distance & 10.37 & 26.57 & 18.47 \\
\hline $0.82 *$ Average distance & 14.50 & 17.33 & 15.92 \\
\hline $0.825^{*}$ Average distance & $\mathbf{1 5 . 7 5}$ & $\mathbf{1 5 . 7 5}$ & $\mathbf{1 5 . 7 5}$ \\
\hline $0.83^{*}$ Average distance & 16.93 & 15.16 & 16.05 \\
\hline $0.85^{*}$ Average distance & 20.27 & 12.75 & 16.51 \\
\hline
\end{tabular}

\section{EXPERIMENTAL SETTINGS}

To evaluate the proposed systems, three well-known error measures, specifically AER (Average Error Rate), FAR (False Acceptance Rate) and FRR (False Rejection Rate) used in many papers in the literature [13] are utilized. AER is the average of FAR and FRR. The AER is generally adopted as a unique measure for characterizing the performance level of biometric systems, and it indicates the security level provided by the biometric system.

For the experiments, our own Bangla database described in Section IV was used. We trained the system with a set of 12 genuine signatures of each individual (class). Then a distance map using the Euclidian distances between all 12 genuine signatures of each class (121 distances) were calculated. The average distance of all 121 distances for each class was found. The average distance of each class was considered as the acceptance/rejection threshold of that class. To get the minimum AER, we empirically found that when FAR and FRR were equal, then the AER was at a minimum. So, we adjusted the acceptance/rejection threshold based on the average distance of each reference class. The minimum AER was desired in the experimental method and it is achieved at the level where the other two error rates (FAR and FRR) were the same. Therefore, the equal values of FAR and FRR shown in Table IV and Table $\mathrm{V}$ are considered to find the least value of AER.

The variations of FAR and FRR using contour features for various thresholds on the Bangla database is shown in Table III. The test set consists of the remaining samples of genuine signatures and all the forged signatures. If the minimum distance between a test sample and the trained samples of a reference class is less than the predefined acceptance/rejection threshold of the reference class, then the test signature is considered as authentic, otherwise it is considered as a forgery.

\section{RESULTS AND DISCUSSION}

As mentioned earlier, the variations of FAR and FRR using contour features for various thresholds on the Bangla database are shown in Table III. Here values of FAR, FRR and AER are shown for five different threshold values. FAR, FRR and AER values obtained from three different features on the Bangla signature dataset are provided in Table IV.

The graphical representation of different values of AER obtained from different features on the Bangla database is also shown in Fig. 9. We measured the verification performance in terms of the commonly used average error rate (AER). From the results shown in Table IV, it can be noted that the best results were obtained when employing the contour features of signature images. However, undersampled bitmap features also provided good results, with only a very small number of features as compared to contour features.

TABLE IV

Verification PERFoRMANCE ON THE BANGLA DATASET FOR 3 DifFERENT FEATURE SETS

\begin{tabular}{|l|l|l|l|}
\hline Feature set & $\begin{array}{l}\text { FAR } \\
\text { (\%) }\end{array}$ & $\begin{array}{c}\text { FRR } \\
(\%)\end{array}$ & $\begin{array}{c}\text { AER } \\
(\%)\end{array}$ \\
\hline $\begin{array}{l}\text { 80 dimensional features } \\
\text { extracted based on } \\
\text { undersampled bitmaps }\end{array}$ & 20.16 & 20.16 & 20.16 \\
\hline $\begin{array}{l}\text { 40 dimensional features } \\
\text { extracted based on } \\
\text { intersection and end } \\
\text { points }\end{array}$ & 32.23 & 32.23 & 32.23 \\
\hline $\begin{array}{l}\text { 320 dimensional features } \\
\text { extracted based on } \\
\text { contour information }\end{array}$ & $\mathbf{1 5 . 7 5}$ & $\mathbf{1 5 . 7 5}$ & $\mathbf{1 5 . 7 5}$ \\
\hline
\end{tabular}


For a comparative study about the performance of these three features, we also computed FAR, FRR and AER values of the proposed model using 100 sets of the GPDS [7] dataset, and the results are provided in Table V.

From Table IV and Table V it can be seen that contourbased features outperform the other two features for both Bangla and the GPDS signature datasets. However, to get a comparative idea between Bangla and English signature databases, the AER values in the GPDS dataset (Table V) are higher than the AER values in the Bangla dataset. This is because, most of the signature samples in Bangla dataset are textual signatures whereas in the GPDS dataset, most of the samples are composed of lines and curved shape patterns, and the features studied in this research work are mainly dedicated to textual patterns. It may be noted that the FAR, FRR and AER are equal in our experimental results because of our threshold selection as discussed in Section IV. The graphical representation of different values of AER obtained from different features using the GPDS database is also shown in Fig. 10.

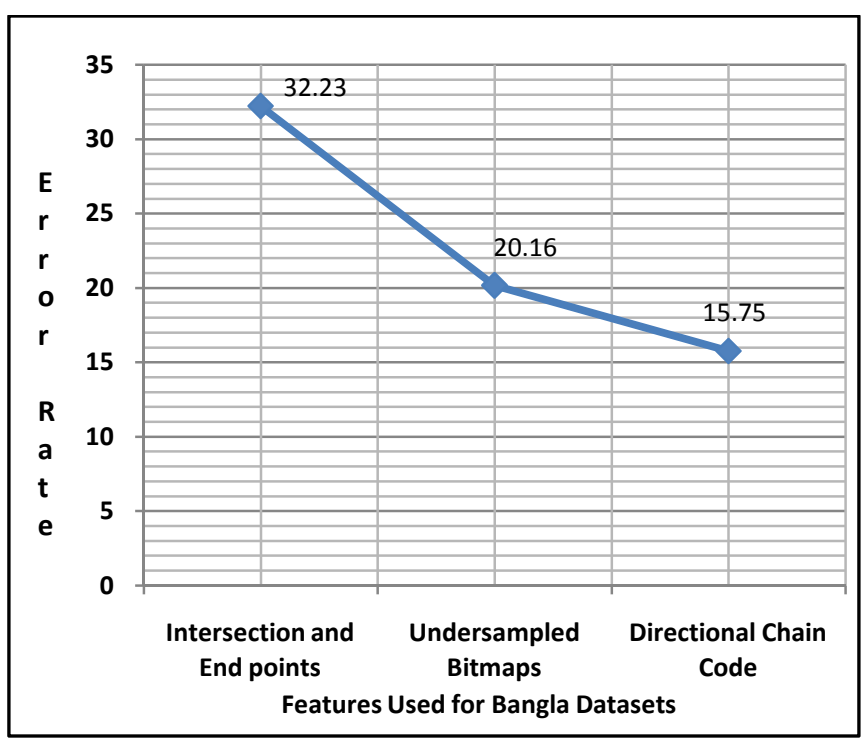

Fig. 9. Representation of average error rate obtained using three different features on the Bangla dataset.

TABLE V

Verification PERFormance ON THE GPDS Dataset For 3 Different FeAture SeTS

\begin{tabular}{|l|l|l|l|}
\hline Feature set & $\begin{array}{l}\text { FAR } \\
\text { (\%) }\end{array}$ & $\begin{array}{l}\text { FRR } \\
\text { (\%) }\end{array}$ & $\begin{array}{l}\text { AER } \\
(\mathbf{\%})\end{array}$ \\
\hline $\begin{array}{l}\text { 80 dimensional features } \\
\text { extracted based on } \\
\text { undersampled bitmaps }\end{array}$ & $\mathbf{2 3 . 5 7}$ & $\mathbf{2 3 . 5 7}$ & $\mathbf{2 3 . 5 7}$ \\
\hline $\begin{array}{l}40 \text { dimensional features } \\
\text { extracted based on } \\
\text { intersection and end } \\
\text { points }\end{array}$ & 33.89 & 33.89 & 33.89 \\
\hline $\begin{array}{l}320 \text { dimensional features } \\
\text { extracted based on } \\
\text { contour information }\end{array}$ & 23.33 & 23.33 & 23.33 \\
\hline
\end{tabular}

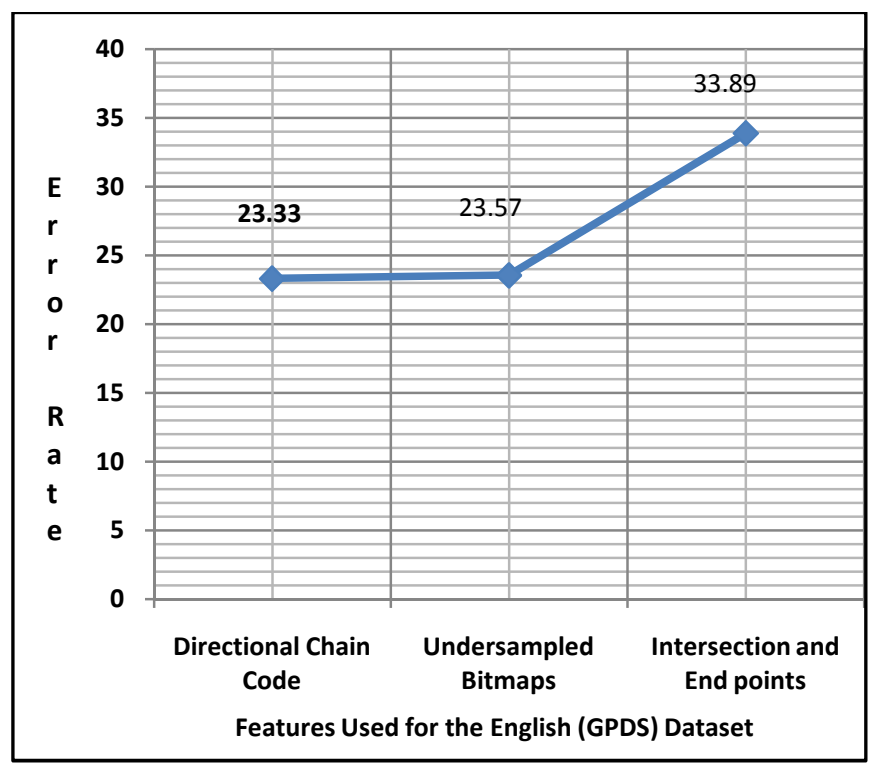

Fig. 10. Representation of average error rate obtained using three different features using the GPDS dataset.

\section{CONCLUSIONS AND Future WorK}

This paper demonstrates an investigation of the excellent performance of threshold-based signature verification technique involving Bangla off-line signatures. This novel approach for signature verification employed a large Bangla off-line signature dataset, providing a substantial contribution to the field of signature verification.

Three different feature sets (under-sampled bitmap feature, intersection/endpoint feature and directional chain code features) are used here to perform a comparative study on the Bangla signature dataset. To the best of our knowledge, the abovementioned methods have never been used for the task of Bangla signature verification. Moreover, this scheme of Bangla off-line signature verification is also a new contribution to the field of signature verification. In the near future, we plan to extend our work considering more samples of Bangla off-line signatures. We also plan to make this extended dataset available publicly to researchers.

\section{REFERENCES}

[1] R. Plamondon and G. Lorette, "Automatic Signature Verification and Writer Identification - The State of the Art," Pattern Recognition, vol. 4, no. 2, pp. 107-131, 1989.

[2] E. Justino, E. Bortolozzi, R Sabourin, "Off-line Signature Verification Using HMM for Random, Simple and Skilled Forgeries," Proceedings of 7th in International Conference on Document Analysis and Recognition, pp. 105-110, 2001.

[3] M.D. Garris, "NIST Form-Based Handprint Recognition System", NISTIR 5469, 1994.

[4] E. Alpaydin, C. Kaynak, "Cascading Classifiers", Kybernetika, 34(4), pp. 369-374, 1998.

[5] C. Rafael, R. Gonzalez, E. Woods, Digital Image Processing, Second Edition, Prentice Hall India, 2002.

[6] F. Kimura, T. Wakabayashi, S.Tsuruoka and Y. Miyake, "Improvement of handwritten Japanese character recognition using weighted direction code histogram", Pattern recognition, vol. 30, no. 8, pp. 1329-1337, 1997.

[7] M. A. Ferrer, J. B. Alonso, and C. M. Travieso, "Offline geometric parameters for automatic signature verification using fixed-point 
arithmetic", IEEE trans. on Pattern Analysis and Machine Intelligence, vol. 27, no. 6, pp.993-997, 2005.

[8] S. Pal, V. Nguyen, U. Pal and M. Blumenstein, "Off-line bangla signature verification", In: International Workshop on Document Analysis Systems, pp. 282-286, 2012.

[9] J. Coetzer, B. Herbst, and J. D. Preez, "Off-line signature verification using the discrete radon transform and a hidden markov model", EURASIP Journal on Applied Signal Processing, vol. 4, pp. 559571, 2004.

[10] B. B. Chaudhuri and U. Pal, "An OCR system to read two Indian language scripts: Bangla and Devnagari (Hindi)", Proceedings of $4^{\text {th }}$ International Conference on Document Analysis and Recognition, pp. 1011-1015, 1997.

[11] B. Fang, C.H. Leung, Y.Y. Tang, K.W. Tse, P.C.K. Kwok and Y.K Wong, "Off-line signature verification by the tracking of feature and stroke positions", Pattern Recognition, vol. 36, pp. 91-101, 2003.

[12] S. Chen, and S. Srihari, "Use of Exterior Contour and Shape Features in Off-line Signature Verification", Proceedings of $8^{\text {th }}$ International Conference on Document Analysis and Recognition, pp. 1280-1284, 2005.

[13] D. Impedovo, G. Pirlo, “ Automatic signature verification: The state of the art", IEEE Transactions on Systems, Man, and Cybernetics part-C, vol. 38, no. 5, pp. 609-635, 2008.

[14] J. Wen, B. Fang, Y. Y. Tang, T. P. Zhang, and H. X. Chen, "Offline Signature Verification Based on the Gabor Transform," Proceedings of the International Conference on Wavelet Analysis and Pattern
Recognition, pp 1173 - 1176, 2007

[15] A. C. Ramachandra, K. Pavithra, K. Yashasvini, K.B.Raja, K.R. Venugopal and L. M. Patnaik, "Offline Signature Authentication using Cross - validated Graph Matching," Proceedings of the second Bangalore Annual Compute Conference, ACM, Article no. 7, 2009.

[16] J. A. Mahar, M. H. Mahar and M. K. Khan, "Comparative Study of Feature Extraction for Off-Line Signature Verification," Second International Conference on Emerging Technologies, pp. 115-120, 2006.

[17] S. Pal, U. Pal and M. Blumenstein, "Off-line English and Chinese Signature Identification Using Foreground and Background Features", In Proc. of IJCNN Special Session on Machine Learning for Computer Vision at 2012 IEEE World Congress on Computational Intelligence, WCCI 2012, pp. 1-7, 2012.

[18] S. Pal, A. Alaei, U. Pal and M. Blumenstein, "Off-line Signature Verification based on Foreground and Background information", Proceedings of International Conference on Digital Image Computing Techniques and Applications, pp. 672-677, 2011.

[19] B. B. Chaudhuri and U. Pal, "An OCR system to read two Indian language scripts: Bangla and Devnagari (Hindi)", Proceedings of $4^{\text {th }}$ International Conference on Document Analysis and Recognition, pp. 1011-1015, 1997.

[20] S. Pal, M. Blumenstein and U. Pal, "Hindi off-line signature verification", In: International Conference on Frontiers in Handwriting Recognition, pp. 371-375, 2012. 